\title{
Utilizing Existing Commercial Geostationary Earth Orbit Fixed Satellite Services for Low Earth Orbit Satellite Communication Relays with Earth
}

Brian Kopp, Jonathan Harris, and Caio Lauand

Electrical Engineering Department, University of North Florida, Jacksonville, Florida.

\section{ABSTRACT}

There are only a few ways that a low Earth orbit (LEO) satellite can communicate with Earth without a ground station network. A LEO satellite can link with other LEO satellites establishing a mesh network that connects with Earth, or LEO satellites can relay their traffic through one of only a few specialized existing or planned geostationary Earth orbit (GEO) or medium Earth orbit (MEO) relay satellites such as the Tracking and Data Relay Satellite System (TDRSS) and Audacy. Unfortunately, there is a real possibility that these limited choices for satellite communications will not accommodate all of the anticipated growth in LEO satellite deployments that are forecast for the next 10 years. The less efficient direct LEO to Earth communications link method will be used unless another solution can be found. A new option may be to leverage the on-orbit commercial GEO satellites that already support the fixed satellite services (FSSs) market. In this scenario, a LEO satellite would relay its communications through the GEO satellite and down to an Earth station, much the same way terrestrial very small aperture terminals, cruise ships, or jet airliners do today. Utilizing several of these commercial GEO satellites, a LEO satellite could communicate with Earth during a large portion of each orbit. There are some challenges with such a scheme, including regulatory hurdles, but if they could be overcome a significant resource of satellite communication services could be engaged to support the future growth of the satellite industry. Several new LEO to GEO relay methods have been proposed or are in development, requiring the deployment of new GEO relay satellites, but this article discusses the idea of utilizing the hundreds of existing FSS GEO satellites operating in $C, K u$, and $K a$ band to leverage an existing resource for satellite communications with LEO satellites.
Keywords: satellite communications, low Earth orbit, geostationary Earth orbit, fixed satellite services, small satellites, tracking and data relay satellites

\section{BACKGROUND}

he small satellite market is growing quickly. Just a few years ago, in 2008, approximately 15 satellites weighing less than 50 kilogram $(\mathrm{kg})$ were launched into orbit around the Earth. ${ }^{1}$ In 2017, a record year for small satellite launches, that number jumped to $300 .{ }^{1}$ More recently, in the first quarter of 2018, 62 small satellites were launched. ${ }^{2}$ Looking forward, one industry estimate that considered small satellites with masses up to $500 \mathrm{~kg}$ expects the average number of launches per year to reach 540 by $2022 .{ }^{3}$ Owing to one planned very large constellation, the following 5 years, through 2027, should see an average number of small satellites launches per year numbering $850 .{ }^{3}$ It should be noted that the majority of planned small satellites, $82 \%$ by one estimate, will be in low Earth orbit (LEO). ${ }^{3}$

There is one thing all of these small satellites need that is a challenge to provide: communications with the Earth. The satellite's mission always requires some transfer of information either to or from the ground. Achieving this link has involved various technologies for the past 60 years, from the mid-air recovery of film canisters ejected in space by CORONA reconnaissance satellites in $1960^{4}$ to the use of Earth-directed laser communications by even small cube satellites, demonstrated in 2018. ${ }^{5}$ However, today, wireless radio communications are the principle technology used for linking small satellites to the ground.

\section{COMMUNICATIONS THROUGH DIRECT GROUND LINK}

For LEO satellites, the use of radio communications usually involves a direct link to the ground using microwave or millimeter wave radio transmissions. Unfortunately, the view of

(c) Brian Kopp et al. 2019; Published by Mary Ann Liebert, Inc. This Open Access article is distributed under the terms of the Creative Commons License (http:// creativecommons.org/licenses/by/4.0), which permits unrestricted use, distribution, and reproduction in any medium, provided the original work is properly cited. 


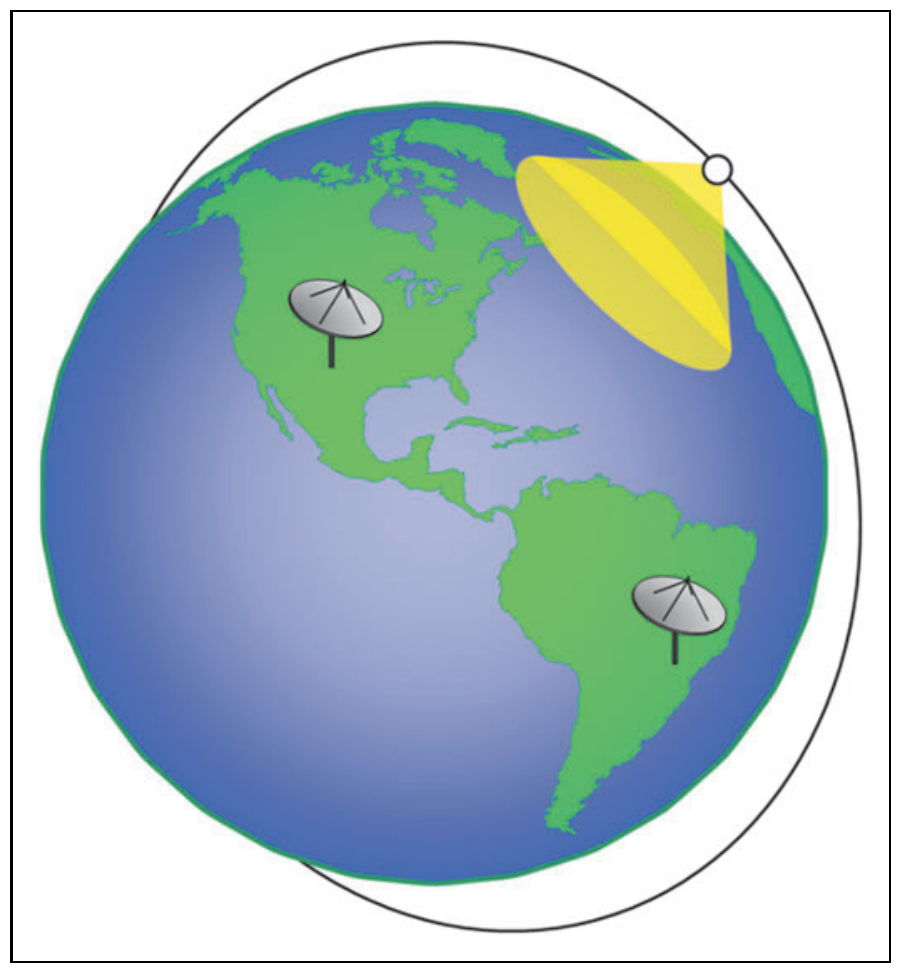

Fig. 1. LEO satellite Earth visibility. LEO, low Earth orbit. the Earth from LEO is only a few thousand miles wide. As a result, a LEO satellite may only occasionally pass over an area of the Earth where an Earth station is located, as is shown in Figure 1. In Figure 2 the ground track of an example LEO satellite in an approximate inclined prograde orbit of $50^{\circ}$ is shown.

Depending on the LEO satellite's altitude, an orbit may take 90 to $100 \mathrm{~min}$ to complete. Each orbit precesses around the Earth as the Earth turns underneath. This means that an Earth station in the mid-latitudes will be in view of the station on 1 or 2 successive orbits, approximately every $12 \mathrm{~h}$. During each fly over of the Earth station, a visibility window of $\sim 5$ to $10 \mathrm{~min}$ is available for the LEO satellite to transfer data to the Earth station. Every $24 \mathrm{~h}$ the LEO satellite would have an accumulative data transfer window of $\sim 30$ min to transfer all of its mission data to one Earth station.

Unlike the mentioned example of a lower inclined orbit that is favored by larger LEO satellites such as the International Space Station or the Hubble Space Telescope, many Earth observing LEO satellites use a polar orbit to trace a path over the entire Earth and also to often take advantage of a unique orbit that is Sun synchronous. A Sun synchronous orbit can be used to keep the LEO satellite continuously in view of the Sun, observing the Earth only in daylight and utilizing continuous solar power. An example ground track for a polar orbiting LEO satellite, where the track reaches the Earth's poles, is shown in Figure 3.

With regard to Earth station communications, 1 advantage for polar orbiting LEO satellites is that Earth stations located

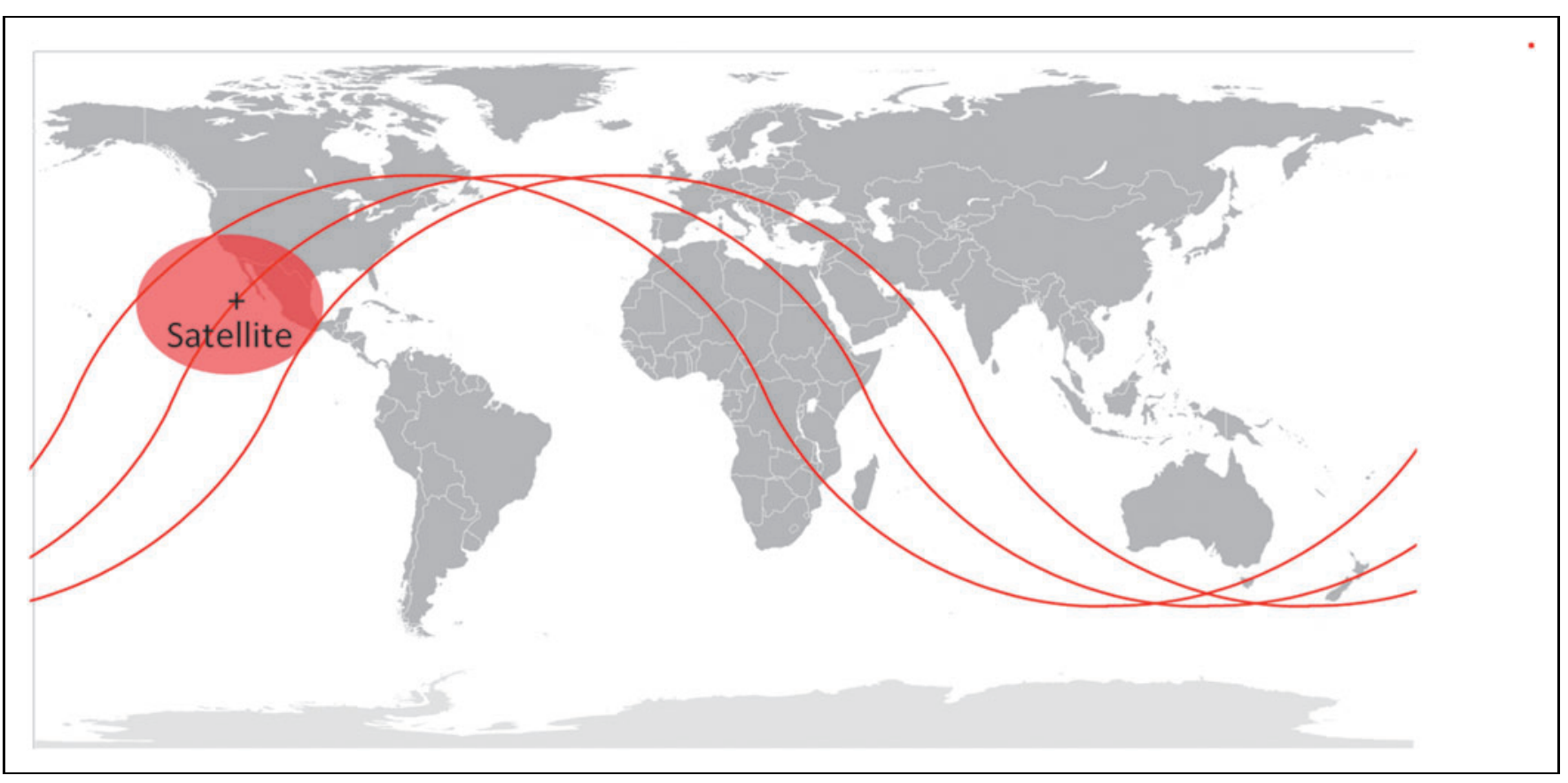

Fig. 2. The ground track of a $50^{\circ}$ inclined LEO satellite. 


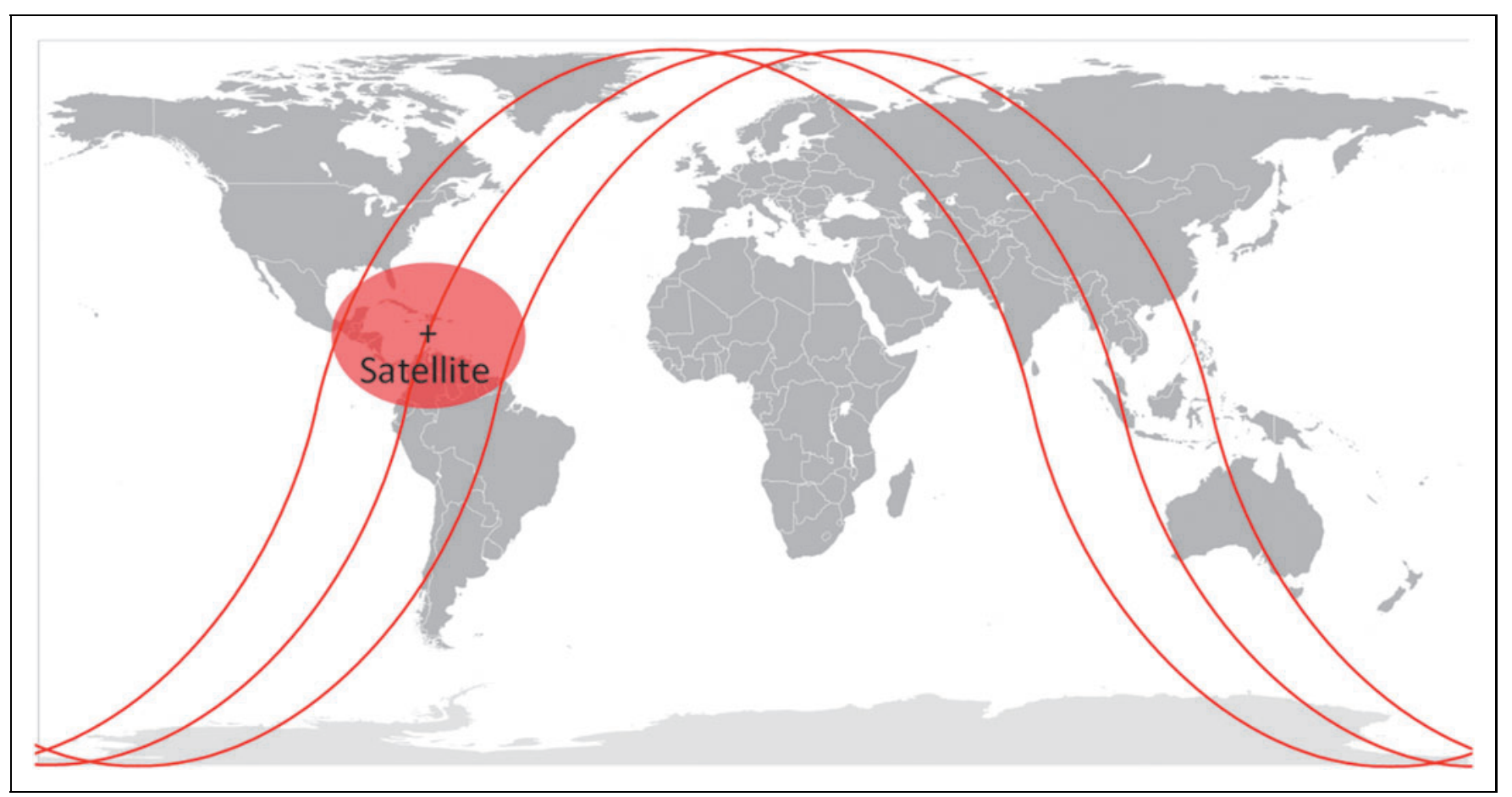

Fig. 3. The ground track of a polar orbiting LEO.

near the Earth's poles can have visibility of LEO satellites on 3 or 4 successive orbits, which is at least twice the number of passes that any mid-latitude Earth stations can see the same satellite. This advantage occurs because the polar satellite's trajectory passes near the poles on every orbit.

The limitation on Earth station visibility means that the LEO satellite has opportunities to transfer data directly to Earth during only a fraction of each 24 -h period. However, there are additional impacts from this restriction on ground communications availability. Having only infrequent Earth station coverage puts a limitation on the timeliness of data that can be transferred. For example, when emergency communications are provided through a satellite for a person on the Earth who activates an emergency position indicating radio beacons, $\sim 10 \%$ of those emergency distress transmissions are received only by Search And Rescue SATellites (SARSATs) in LEO orbit. $^{6}$ The message is stored on the LEO satellite until it passes over an Earth station. Using only LEO SARSATs delays the mobilization of rescue services by an average of $49 \mathrm{~min}^{6}{ }^{6}$

There can be additional impacts for more complicated applications such as mobile voice telephony through satellite. If the telephone call can only be made through the LEO satellite during a pass over an Earth station, then making a satellite phone call would require the customer and the Earth station to both be in view of the satellite during the call. This coverage limitation can be mitigated but it requires significant design changes.
Two prominent vendors of satellite-based voice telephony that use LEO satellites are Iridium and Globalstar and they solve this challenge of Earth station communications in different ways. Iridium uses a fleet of more than 60 polar orbiting LEO satellites to ensure customers can see a satellite. Iridium added inter-satellite microwave linking to each satellite, to create a mesh network of satellites, ensuring that a phone call can always link to a satellite over an Earth station that has a telephone network connection. Globalstar uses a fleet of 24 LEO satellites orbiting in a $52^{\circ}$ inclined orbit to ensure customer coverage to a significant portion of the Earth but operates a global network of approximately 27 Earth stations to provide the necessary links to the ground to complete each call. Both Iridium and Globalstar are considered mobile satellite service (MSS) providers. They market lower speed data and voice connections to mobile and remotely located stationary customers on the Earth.

\section{COMMUNICATIONS THROUGH LEO MESH NETWORKS}

There are communication alternatives for LEO satellites to consider instead of using only a direct link to Earth stations as the satellite flies over it. The Iridium network demonstrates one of these alternatives. Their space-linked satellites do require complex satellite communications hardware and software to deploy and operate the network. Each Iridium satellite operates four intersatellite links in the Ka band spectrum. 
Since these sophisticated satellites are on orbit and not easy to repair, the technology must be especially reliable. This makes the space-linked solution to the satellite communications an expensive one. Nevertheless, when compared with operating a network of ground stations, the space-linked solution can be viable under the right conditions.

An exciting newer technology that may impact the business model for communications through a mesh of LEO satellites is the introduction of optical-based intersatellite linking (ISL). Free-space optical communications offer much higher data rates than traditional microwave communications. In addition, propagation conditions for free-space optics are ideal for LEO to LEO ISL, provided both satellites have line-of-sight to each other above the Earth's atmosphere. Attitude control for optical target focusing and Doppler mitigation are challenges to this approach, but they are surmountable.

Although free-space optical communications are certainly of interest for LEO to LEO ISL, there is a significant technical challenge to using free-space optical communications to link the LEO mesh network to Earth. To reliably transmit higher data rates to the Earth through direct optical path requires multiple redundant Earth station links to overcome inclement weather at specific Earth stations that cannot receive optical communications. Nevertheless, National Atmospheric and Space Administration (NASA) is hoping to deploy an operational optical communications relaying geostationary Earth orbit (GEO) satellite by $2025^{7}$ and the commercial satellite market is on the verge of deploying optical communications. ${ }^{8}$

Another alternative solution for LEO satellite communications with Earth, analyzed by Horan, ${ }^{9}$ considers operating the LEOs as customers on the networks established by MSS providers such as Iridium and Globalstar. This would, in effect, create a new class of customer for these MSS networks. Careful planning for matching orbits could improve the amount of time that a link to the ground is available.

Horan identifies the challenges for such a solution as being regulatory and access related. The regulatory issue is that this solution includes a new use of existing MSS radio frequency spectrum to create the link between customer LEO satellites and the MSS network satellites. Today this spectrum can only be used for links between space and the Earth, and not for space-to-space ISL. Global regulatory permissions would be needed to establish the use of this MSS spectrum for space-to-space customer links. The access issue identified by Horan involves the changes to their operations that the MSS vendor must make to accept LEO-based satellite customers instead of Earth-based handset customers. A business case must be made to justify the cost of any required modifications to the MSS vendor network, including technical, operational, and management and billing modifications.

\section{COMMUNICATIONS THROUGH SPECIALIZED GEO AND MEDIUM EARTH ORBIT RELAY SATELLITES}

A third alternative solution for the communications challenge facing small satellites involves the use of specially designed GEO or medium Earth orbit (MEO) satellites acting as relays between LEO satellites and Earth stations. This alternative solution has been used for more than 35 years by the U. S. government. The NASA Tracking and Data Relay Satellite System (TDRSS) operates 6 active GEO satellites that can each relay communications between LEOs and the Earth. The satellites are positioned at 3 strategic longitudes to maximize global LEO coverage. An example of a TDRSS constellation is shown in Figure 4 and demonstrates that continuous global coverage for LEO satellites is possible.

Today the TDRSS is used by NASA as well as other federal agencies. The system has specific requirements for communications equipment that must be installed on the user's platform, which can be a LEO satellite, space station, aircraft, etc. The NASA Goddard Space Flight Center, which operates the TDRSS spacecraft, has studied how to adapt TDRSS for small satellites, ${ }^{10}$ and the third generation of satellites in the network now include Ka band services as well as return-link demand access services (DASs) that are offered on a shared first-come first served basis, but that are more useful for occasional and short-notice LEO satellite communication services.

In general, the TDRSS is a schedule driven service with users supported according to a priority list. Users are required to be flexible with regard to a window of support time during which a schedule order (SHO) for their communications needs will be executed. A SHO can cover many minutes of satellite communications support from TDRSS. The rates for the various types of services on TDRSS are published by NASA.${ }^{11}$ One minute of the fastest 2-way service on TDRSS, called single access service, can typically support communication speeds on the order of 50 to 300 Mega-bits-per-second (Mbps) and costs 136.37 U. S. dollars (USD). If it were possible to use this service continuously for a month at that rate, it would cost 5.9 million USD.

A less expensive TDRSS service, called multiple access (MA) service, that can typically support communication speeds on the order of 6 Mbps costs 34.62 USD per minute, when used in a 2-way configuration. For a month of 2-way MA service, the cost would be 1.5 million USD. The newest service on TDRSS is DAS, a form of random access service. As already discussed, this is a shared service so it is difficult to ensure a particular communications event will be supported at the specific desired time. The costs are lower for this service where a single LEO satellite can use the DAS return service for an annual cost of 193,000 USD. It is important to emphasize that DAS does not 


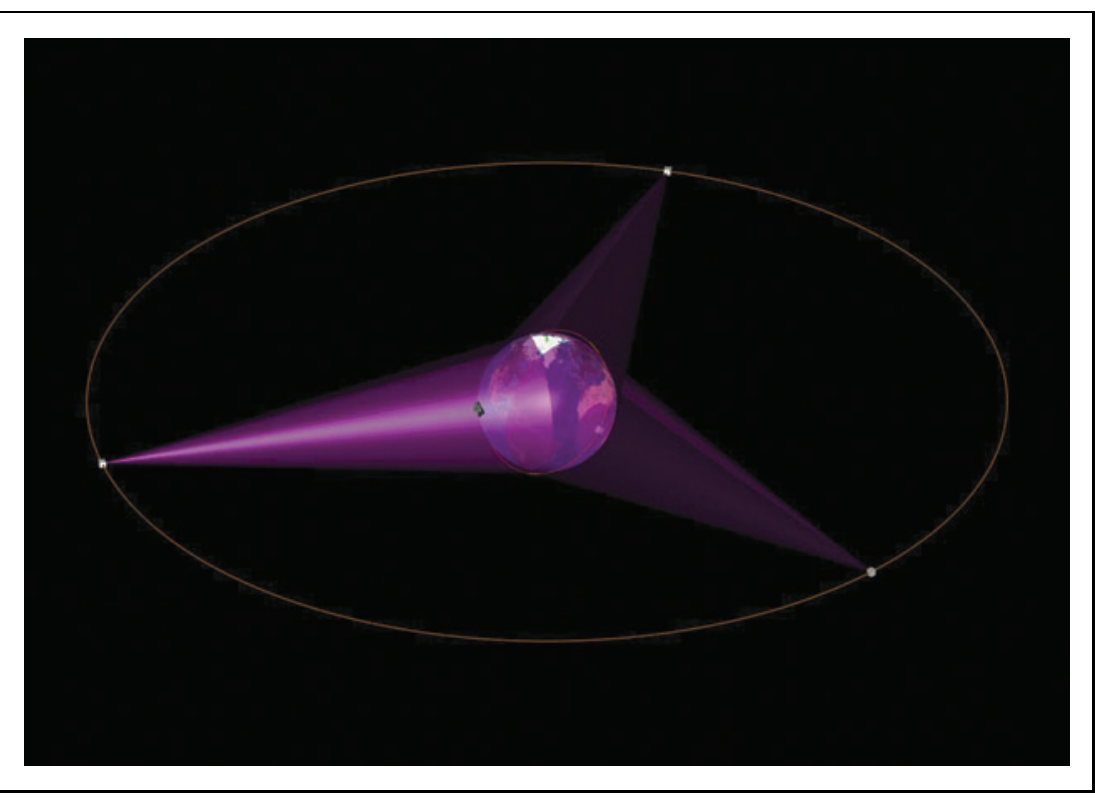

Fig. 4. Example TDRSS constellation. TDRSS, Tracking and Data Relay Satellite System.

include a forward link to send data to the LEO satellite. The DAS can only downlink data from the LEO satellite.

Although the TDRSS has been providing data relay services for government agency LEO satellites since TDRS-A was launched in 1983, the first commercial version of a TDRS-like system was only recently licensed by the Federal Communications Commission (FCC) in the spring of 2018. ${ }^{12}$ Audacy intends to build a network of 3 MEO relay satellites that will use Ka band frequencies allocated for ISL to communicate with customer LEO satellites. These MEO satellites will then relay customer communications to Audacy Earth stations using frequencies allocated for fixed satellite services (FSSs). The Audacy network is a 300 million USD project currently in development and is expected to be deployed by $2020 .{ }^{13}$ Within months of receiving their license from the FCC, Audacy announced that it had secured 100 million USD worth of global commercial agreements to provide relay services when its services begin. ${ }^{14}$

Inmarsat has also proposed that the MSS L-band Earth-tospace and space-to-Earth spectrum be reallocated for ISL. Inmarsat and its partners have developed and tested an L-band LEO satellite terminal that would communicate with the 4 on-orbit Inmarsat-4 GEO satellites. In this scenario, the LEO satellite effectively acts as a Broadband Global Area Network (BGAN) customer and the Inmarsat-4 satellites relay their data to Earth using the Inmarsat BGAN network. ${ }^{15}$ To date, in the United States, the FCC has not ruled on the proposal. ${ }^{16}$ The international regulatory community meets next in November 2019, which may give Inmarsat an opportunity to advance its proposal on the global stage.

There is also recent research in the literature on optimizing new GEO relay satellite and antenna system designs for use with LEO satellites. Knogl ${ }^{17}$ proposed a new GEO relay satellite design that incorporated both novel adaptive modulation and an adaptive multibeam antenna array to support high data rate links on up to 15 LEO satellites simultaneously. Rao ${ }^{18}$ analyzed Ka band antenna beam width and steering requirements to support high data rate ISL.

\section{A NEW ALTERNATIVE: UTILIZING GEO FSSS}

One new alternative communications method that may help small and large constellations of LEO satellites relay their data to Earth is to consider a satellite-based communications service that is already deployed and in use. The FSS is available on several hundred existing GEO satellites around the world, offering a wide range of data speeds from very low speed all the way up to broadband speeds.

Today FSS GEO satellites provide data networking connectivity and dedicated communications links to businesses on the ground, at sea and in the air. Large global FSS vendors include Intelsat, SES, Eutelsat, and Telesat. The FSSs are part of the general satellite services industry. The industry includes chiefly the 3 services FSSs, MSSs, and broadcast satellite services (BSSs), which transmit satellite-based television, radio, and consumer internet services. Many FSS vendors are also BSS vendors. In 2016, the combined satellite services industry was worth almost 128 billion USD $^{19}$ with the BSS sector responsible for the highest revenues.

\section{WHAT FSS HAS TO OFFER LEO SATELLITES}

The GEO satellites working in the FSS industry provide "bent pipe" connectivity to their customers. For instance, an enterprise customer operating a regional or nationwide chain of retail stores may install satellite terminals at each store and connect them through a single satellite uplink connection. On the satellite, the combined signals from all the stores are turned around and transmitted together back to the ground at a satellite Earth station at the enterprise headquarters. The enterprise may communicate with all of its stores using one or more uplink signals that pass though the satellite going in the opposite direction back down to all the store locations.

The "bent pipe" transponder on the satellite has a specific amount of bandwidth that can be subdivided into smaller 
bandwidth segments. In addition, a single segment bandwidth, or channel, can be used by a single carrier signal, referred to as single channel per carrier* or the channel can be shared by many lower speed customers using time division multiple access (TDMA). The former technique delivers a circuit switched connection and the latter technique delivers a packet switched connection. The satellite will usually combine transponder signals into a shaped antenna pattern called a beam that can be directed at a specific region, country, or continent.

If it is desired to take the input from one transponder and connect it to the output of another transponder, this is called "cross-strapping" and it is supported on some FSS satellites. It permits the signals from one beam to be connected with the signals of another beam. In the mentioned example, crossstrapping might permit the headquarters to interface with the satellite on 1 beam in 1 area of the country, while its stores interface with the satellite through several different beams that illuminate other areas of the country. If the beams are operating in different frequency bands, the cross-strapping also translates the band of frequency operation. In this example, it might mean the headquarters operates its link to the satellite in one band and the stores operate their links in another band.

There are 3 frequency bands ${ }^{20, *}$ that are used for FSSs today (with several other higher frequency ranges also allocated but not yet utilized by the industry. The lowest frequency band is called "C-band." The next highest band is "Ku band" and the highest band is "Ka band." The limits of these bands, available for nonfederal users in the United States, are shown in Table 1. In each band, there are different frequency ranges for spaceto-Earth communications and for Earth-to-space communications. The GEO communication satellites that provide FSSs in these bands use a somewhat reduced set of frequency ranges due to various limitations, for instance, sharing the spectrum with non-GEO FSS satellites. However, it is still true that the amount of available spectrum increases with increasing frequency band. The Ka band spectrum available for FSS is almost 2.5 times more than that available to C-band.

There are 2 general types of FSS GEO satellites. The traditional GEO satellite supports perhaps several dozen transponders and may use several antennas or beams, ultimately providing on the order of $1 \mathrm{Gbps}$ of capacity to its customers. The newer type of FSS GEO satellite is the high-throughput satellite (HTS), which can leverage many dozens and even hundreds of $\mathrm{Ku}$ and or $\mathrm{Ka}$ band transponders working through dozens of small beams that

*The bands of interest here as defined by the Institute of Electrical and Electronics Engineers are S Band:2-4 Giga Hertz (GHz), C Band: 4-8 GHz, X Band: 8-12 GHz, Ku Band: $12-18 \mathrm{GHz}, \mathrm{K}$ Band: $18-27 \mathrm{GHz}$, Ka Band: $27-40 \mathrm{GHz}$, and V Band: 4075 $\mathrm{GHz}$

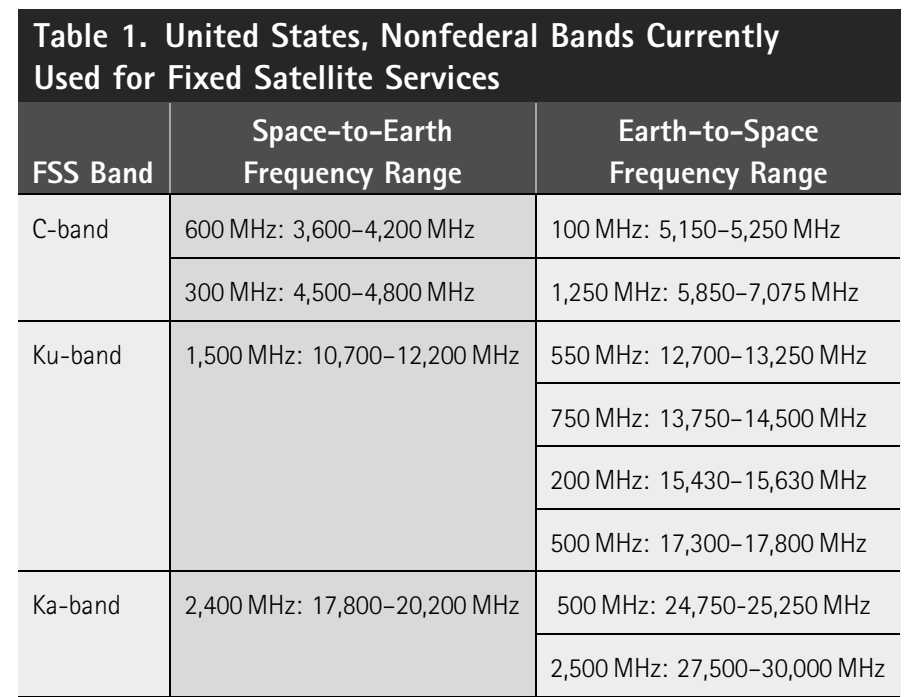

FSSs, fixed satellite services.

reuse frequencies assigned to the satellite much the way the cellular telephone network reuses frequencies in nonadjacent cells. The individual beams typically cover regions as small as a few hundred kilometers across.

One of the most advanced HTSs, the ViaSat 2, launched in June of 2017, uses only Ka band to support a capacity of 260 Gbps and can provide $100 \mathrm{Mbps}$ data service to internet customers. ${ }^{21}$ In September 2017, Intelsat launched the Intelsat 37e satellite, which is the first HTS that can cross-strap transponders from all $3 \mathrm{FSS}$ bands. ${ }^{22}$

To develop an example of how FSS GEO satellites could be used for relaying, consider just the $\mathrm{C}$ band spectrum for an operational scenario. Each LEO satellite would transmit and receive $\mathrm{C}$ band communication signals to and from several FSS GEO satellites. Each of the FSS GEO satellites would operate as a bent pipe relaying the LEO satellite communications to a customer Earth station. As the LEO satellite moved in its orbit, it would move in and out of the coverage of 1 FSS GE0 satellite and on to the next FSS GEO satellite.

Now consider the example $\mathrm{C}$ band FSS GEO satellite coverage maps in Figures 5 through 8. These maps are representative of some of the services available from just 1 FSS vendor. Each coverage map shows the area over which C band FSS is offered. By stitching the four maps together in sequence, it is possible to see how this simple configuration may offer significant orbit coverage for a LEO satellite. The 3 satellites used for the example could be accessed from 3 separate customer Earth stations or leased Earth station services could be retained.

In a second example set, a sequence of FSS GEO satellite coverage maps in the $\mathrm{Ku}$ band spectrum is used. Here in 


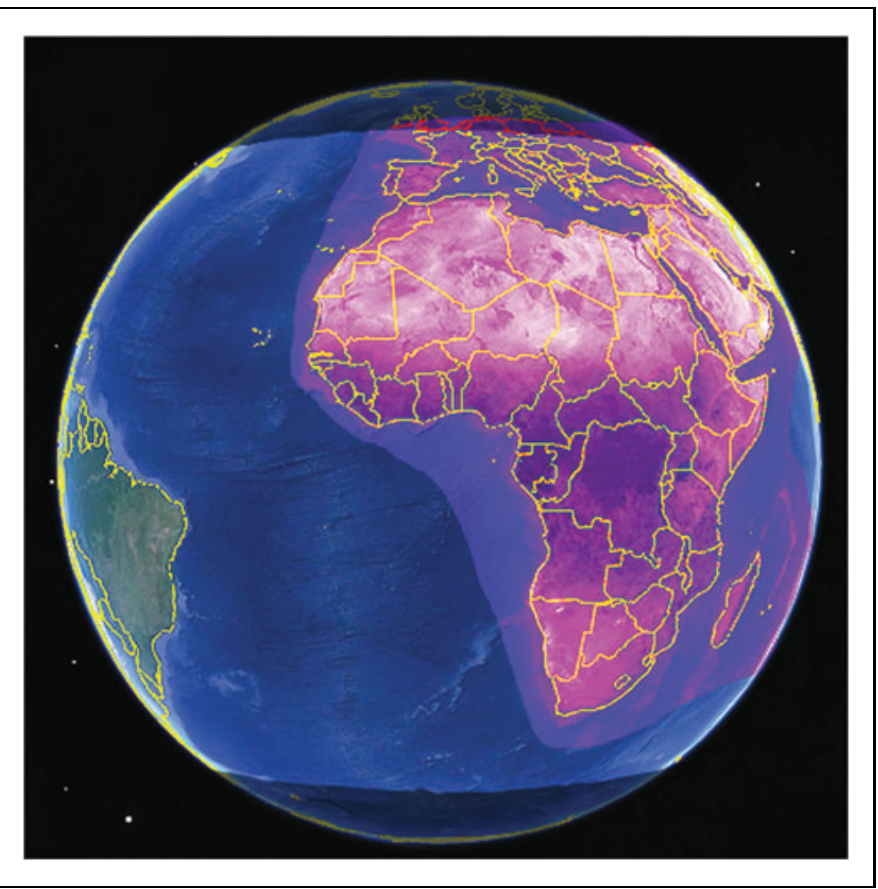

Fig. 5. Example FSS GEO C-band coverage at $0^{\circ}$ longitude. FSSs, fixed satellite services; GEO, geostationary Earth orbit.

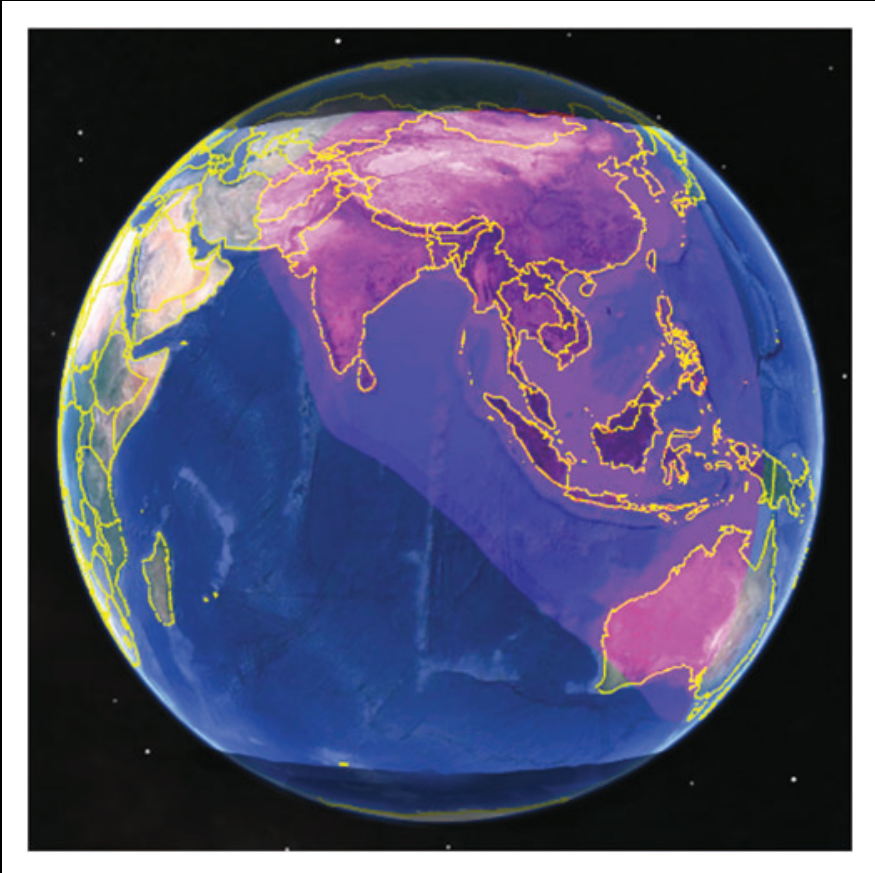

Fig. 6. Example FSSs GEO C-band coverage at $90^{\circ}$ east longitude.

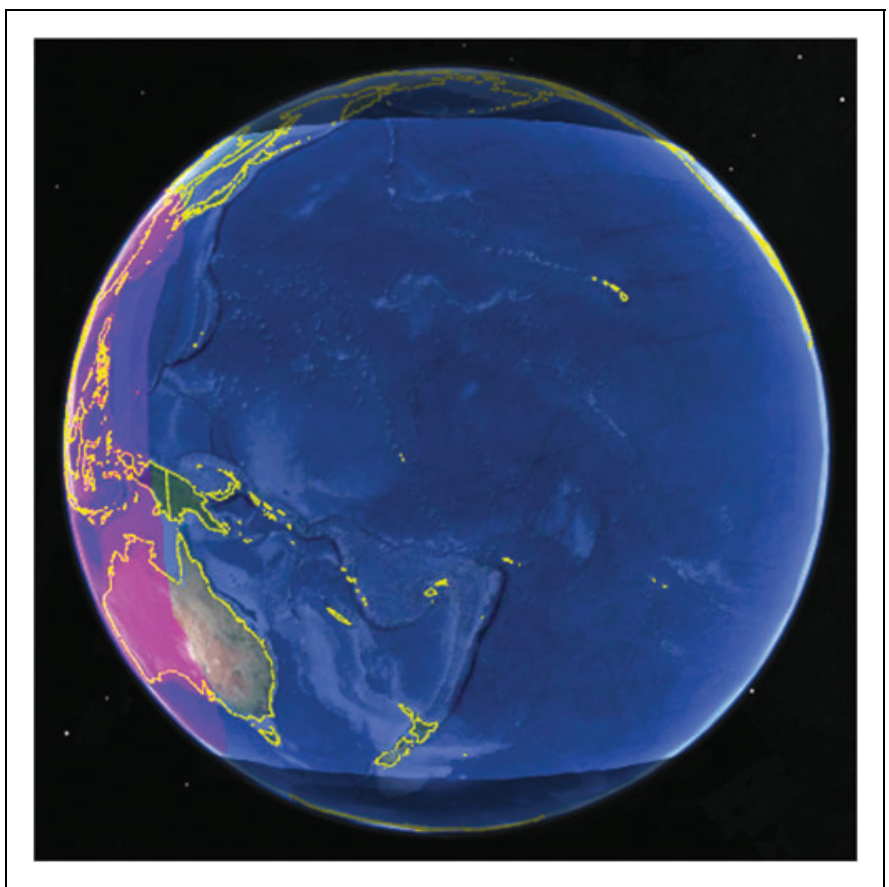

Fig. 7. Example FSSs GEO C-band coverage at $180^{\circ}$ longitude.

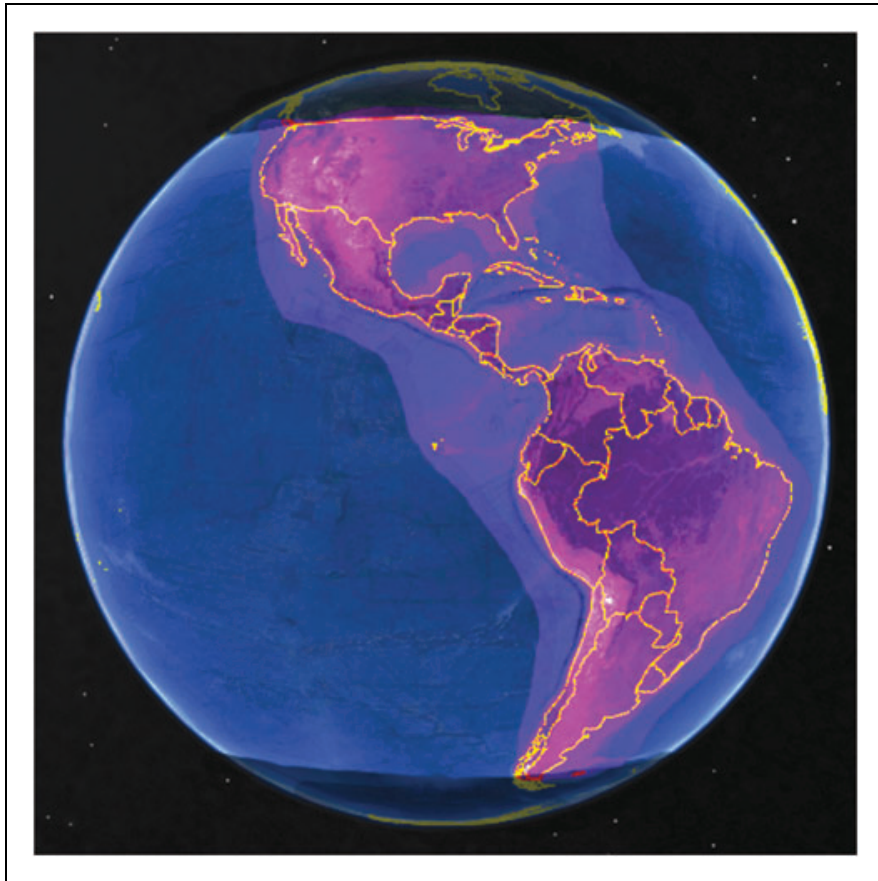

Fig. 8. Example FSSs GEO C-band coverage at $90^{\circ}$ west longitude. 


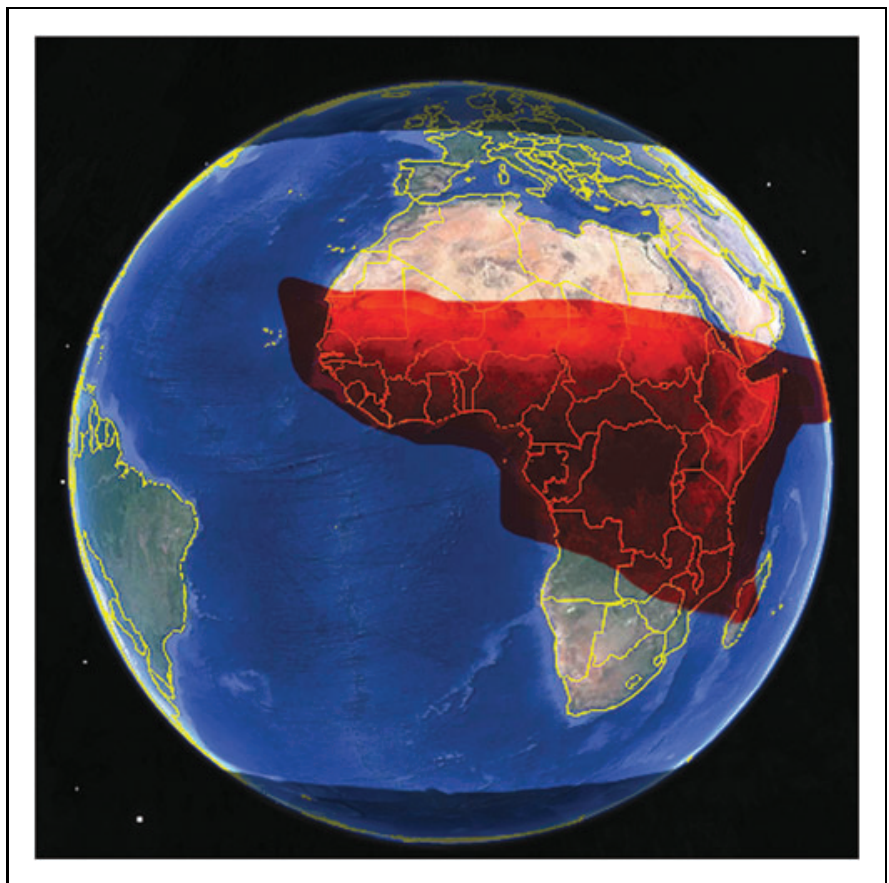

Fig. 9. Example FSSs GEO Ku-band coverage in 1 beam at $0^{\circ}$ longitude.

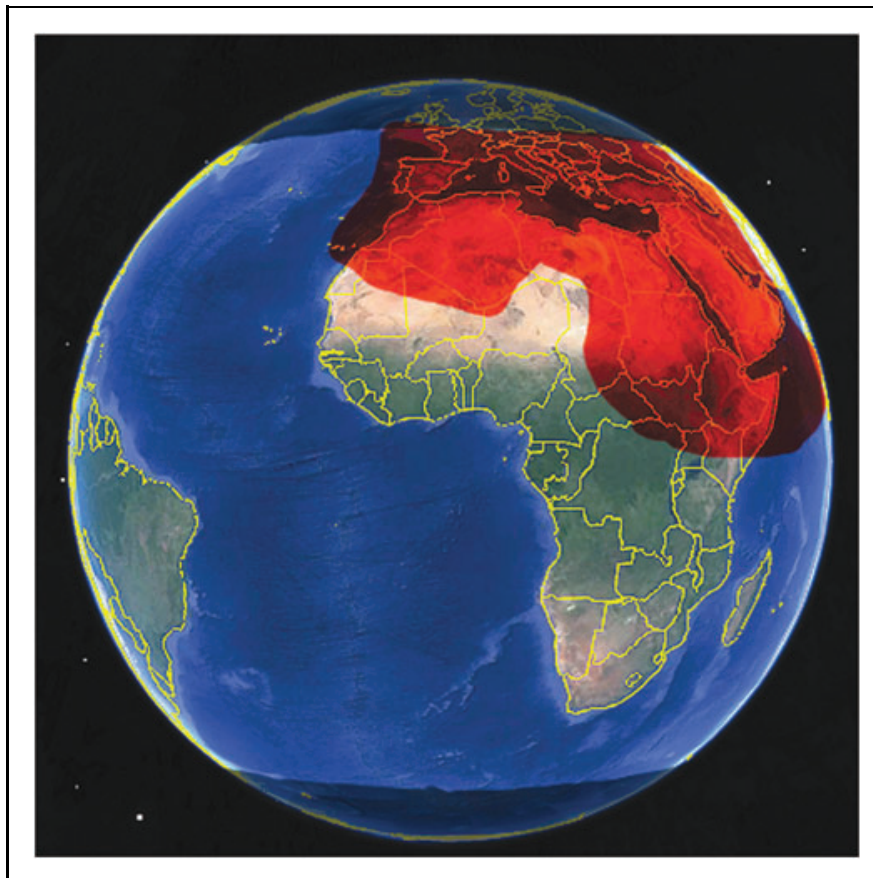

Fig. 10. Example FSSs GEO Ku-band coverage in a second beam at $0^{\circ}$ longitude.

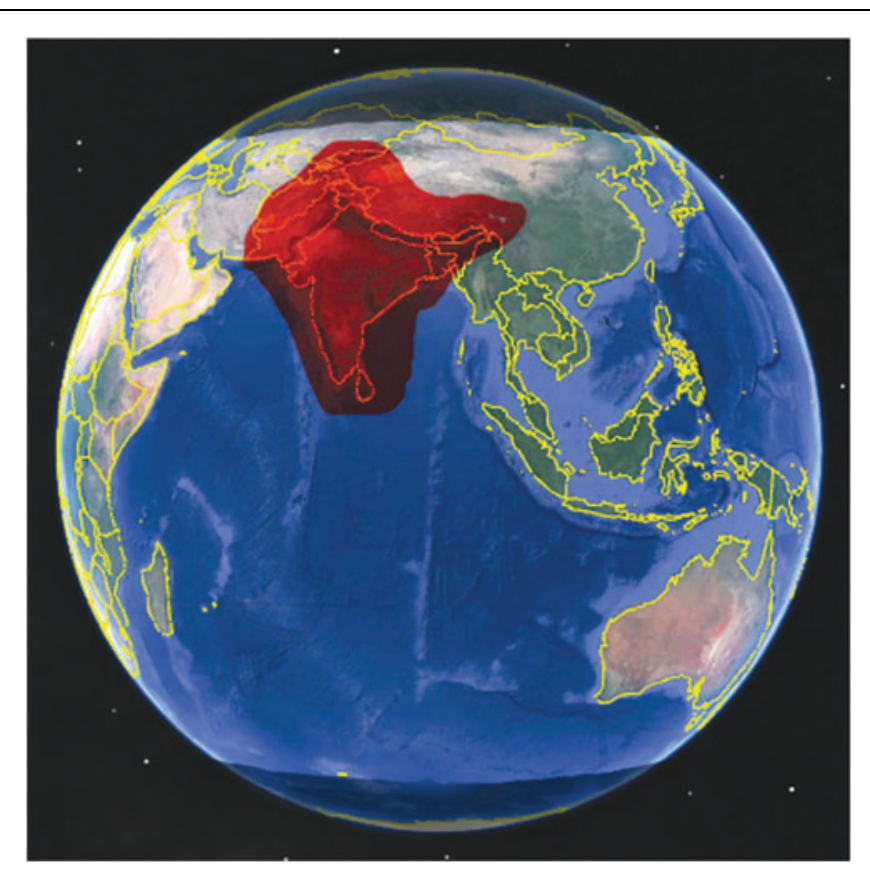

Fig. 11. Example FSSs GEO Ku-band coverage in 1 beam at $90^{\circ}$ east longitude.

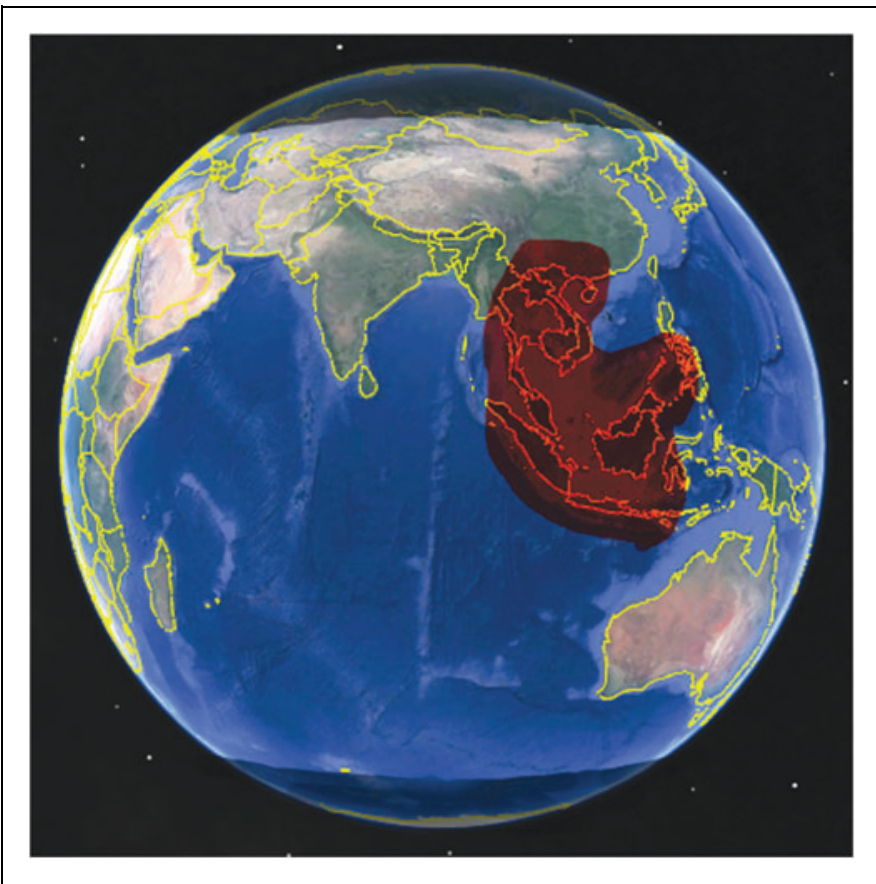

Fig. 12. Example FSSs GEO Ku-band coverage in a second beam at $90^{\circ}$ east longitude. 


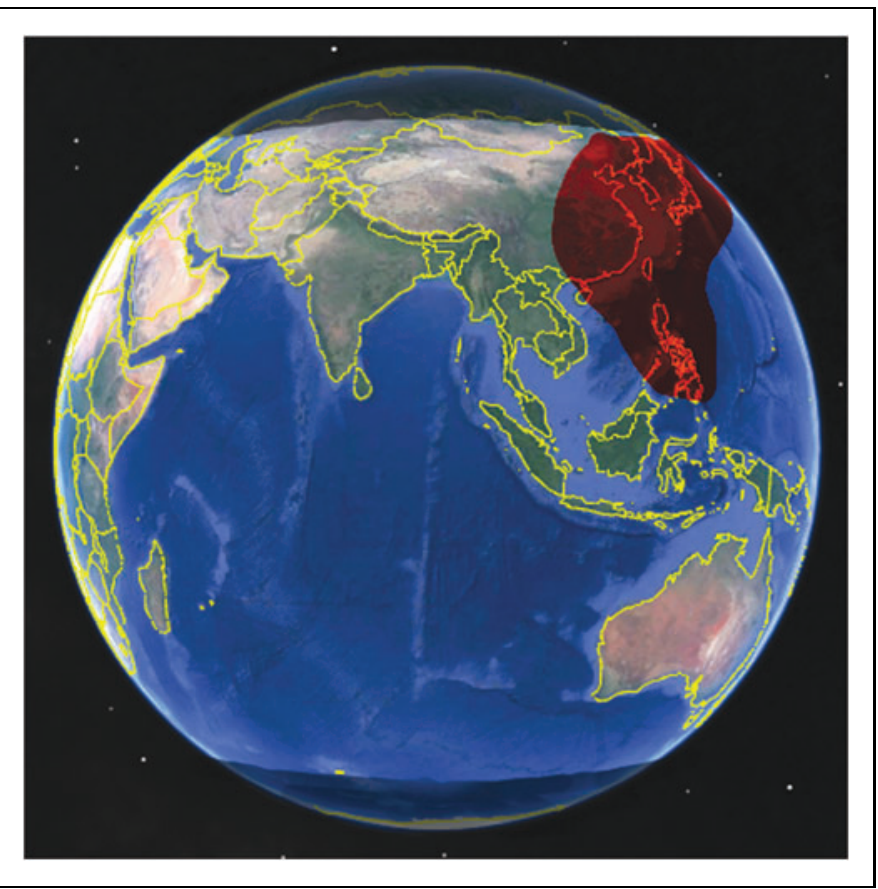

Fig. 13. Example FSSs GEO Ku-band coverage in a third beam at $90^{\circ}$ east longitude.

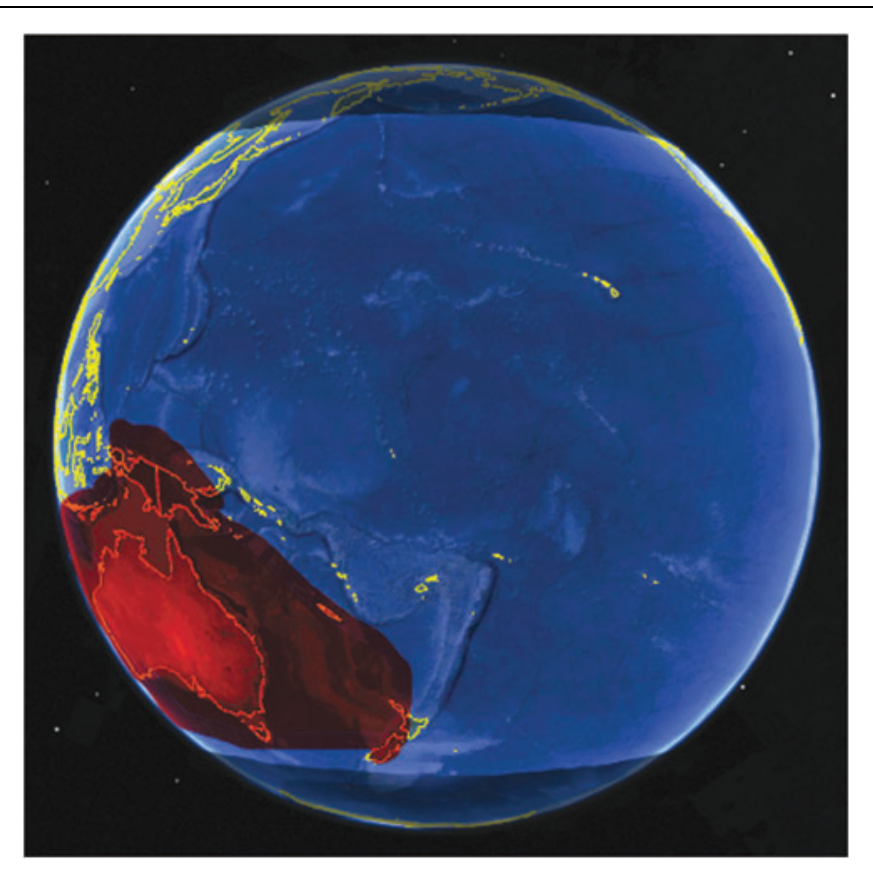

Fig. 14. Example FSSs GEO Ku-band coverage in 1 beam at $180^{\circ}$ longitude.

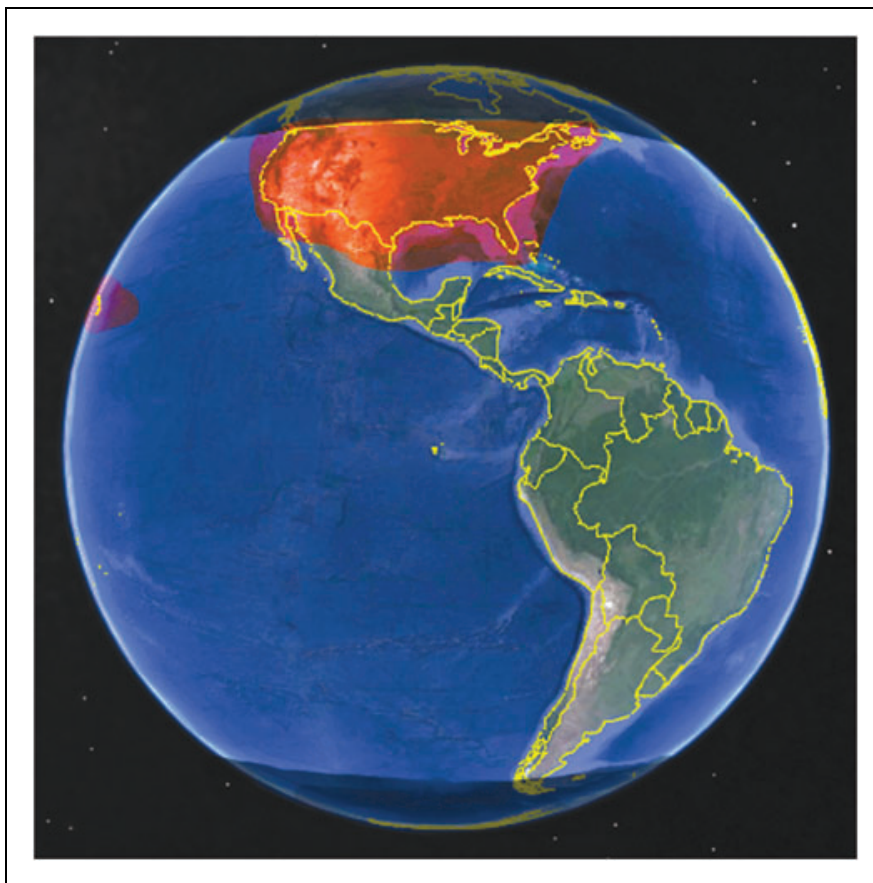

Fig. 15. Example FSSs GEO Ku-band coverage in 1 beam at $270^{\circ}$ longitude.

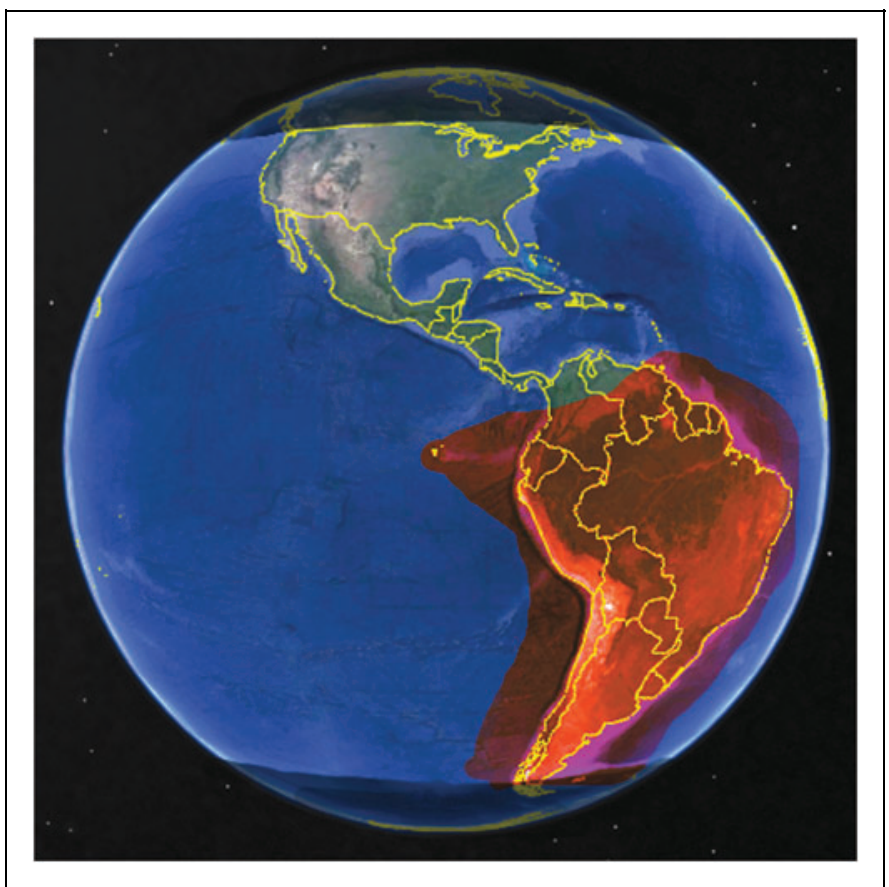

Fig. 16. Example FSSs GEO Ku-band coverage in a second beam at $270^{\circ}$ longitude. 
Figures 9 through 16, the selective $\mathrm{Ku}$ band coverage areas from 8 beams on 4 satellites are used to construct a significant coverage profile for a LEO satellite. Note the coverage areas are smaller and more precisely controlled.

If the third spectrum band available to FSS GEO satellites is considered, Ka band, the coverage beams generally become small spot beams, on the order of hundreds of miles across. Large overlapping patterns of these spot beams make up the $\mathrm{Ka}$ band coverage for a given area, allowing the satellite operator to control more precisely which users are assigned to which beams. The orbital speed of a LEO satellite would suggest that it would spend less than 1 minute in a typical single Ka band spot beam and that it might cross into dozens of beams as it traverses the Ka band coverage area for a specific satellite.

Since the mentioned examples include 3 or 4 FSS GEO satellites, the efficient use of their services must be discussed. The FSS vendor will not, as a rule, sell the full bandwidth of a transponder for only $20 \mathrm{~min}$ out of every $90 \mathrm{~min}$. If FSS GEO relay service is being considered for a single LEO satellite, then a TDMA approach should be considered since it is more appropriate for occasional traffic.

In contrast, if a LEO satellite constellation operator has a significant number of satellites on orbit simultaneously, then controlling an entire FSS GEO satellite transponder or a portion of a transponder's bandwidth may be appropriate since the LEO constellation operator can then dictate the sequencing and even the protocols for FSS GEO satellite relay that will be used. Supporting a larger LEO satellite constellation in this way would ensure that transponder's bandwidth was always supporting some minimum number of the LEO satellites in the constellation, meeting the economic justification for leasing the bandwidth.

\section{THE CHALLENGES TO USING FSS \\ TO RELAY LEO COMMUNICATIONS}

There are several aspects of the FSS GEO satellite relay concept that must be considered to evaluate the feasibility of the scheme. They include both technical and regulatory considerations and some of them present challenges that need to be overcome.

\section{SATELLITE BEAM HAND-OFFS}

The customer base for FSSs is becoming more and more mobile and concepts including communications on the move (COTM) and Earth stations in motion (ESIM) are now part of the lexicon of the satellite communications industry. The most exciting new addition to this mobile customer market is the broadband in-flight service in the commercial and private aviation sectors. Thanks to an FSS connection to the aircraft of several commercial airlines, it is now possible to have an acceptable online experience while traveling across country or even internationally in some areas.

Companies such as Gilat have developed systems for aircraft that use both $\mathrm{Ku}$ band and $\mathrm{Ka}$ band spectrum providing upward of $400 \mathrm{Mbps}$ for passengers to connect to the internet through FSS. ${ }^{23}$ This concept of the mobile customer and now the high-speed mobile airborne customer has meant that the FSS industry has had to adapt its operations to permit the moving customer to traverse between beams. This involves both technical and financial complexities since the customer would like to minimize the downtime during beam transitions and the satellite operator would like to accurately bill the customer for their access.

A LEO satellite moves at $\sim 17,000$ miles per hour (MPH) or 34 times faster than a jet aircraft. Although the FSS industry has seemingly solved the COTM technical and billing challenges for the aviation industry, the higher speed satellite beam hand-offs associated with LEO relay will present a new challenge.

For a circular Ka band spot beam with an example diameter of 350 miles on the Earth, a LEO satellite orbit that splits the spot beam in half will traverse the beam in $\sim 74 \mathrm{~s}$. An orbit that traces a chord across the circular spot beam will obviously experience a shorter spot beam link time. However, if the LEO satellite traverses close to the major axis of an elongated $\mathrm{Ka}$ band spot beam, typically associated with higher latitude coverage, the spot beam link time may be perhaps 2 times longer than the maximum link time for a circular spot beam. An example layout for Ka band spot beams that shows the beam elongation is shown in Figure 17.

\section{SATELLITE FREQUENCY DOPPLER}

The relative motion of the LEO satellite toward and away from the FSS GEO satellite will impose an increase in frequency and then a decrease in frequency on the link between the 2 satellites. For C-band the Doppler frequency offset will be an approximate worst case of $\pm 100 \mathrm{kHz}$. For Ku band, the Doppler frequency offset will increase to a worst case $\pm 450 \mathrm{kHz}$. If Ka band is used, the Doppler frequency offset will be at worst $\pm 750 \mathrm{kHz}$.

Modern satellite communication system architectures can adapt their modems with frequency agile transmitters and receivers that can advance and retard the link frequency to compensate for the computed Doppler change in frequency in real time. This would be a likely requirement for a successful LEO to GEO relay configuration. If the Doppler effect is not compensated for, the LEO satellite would consume valuable GEO satellite transponder bandwidth beyond the edges of the transmission bandwidth to ensure the Doppler shifted signal is relayed without affecting adjacent spectrum licenses. 


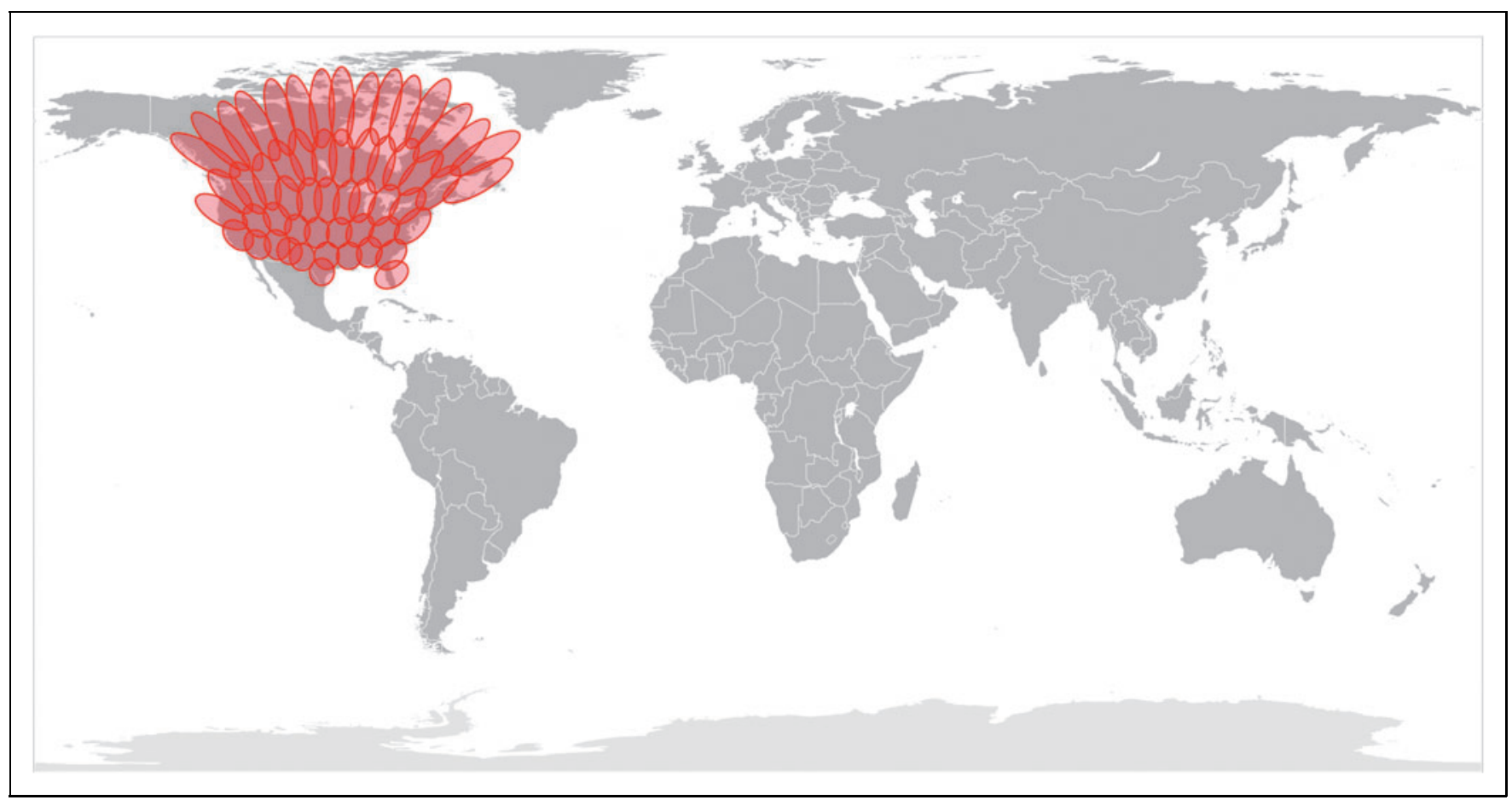

Fig. 17. Example Ka band spot beam coverage layout.

\section{ANTENNA AND TRANSMITTER DESIGN}

The antenna requirements needed for the LEO satellite to utilize the FSSs will be those of a compliant FSS ESIM. The same restrictions on beam width, frequency stability, emission masks, and minimizing interference to nearby GEO satellites will need to be met. The impact on the antenna design will be significant and a stabilized spacecraft platform will likely be necessary. In addition, the larger required antenna size may preclude the smaller form factor satellites from using C-band. New antenna designs that are supporting the COTM market as well as the small satellite industry will help resolve some of the more challenging antenna design issues.

\section{REGULATORY ISSUES}

Currently, LEO satellites are not permitted to communicate with GEO satellites using FSS spectrum. A change to the International Telecommunications Union (ITU) rules and then to the rules of the individual countries involved will need to be made. The change would permit ISL in the FSS bands. Given that FSS is already supporting aviation in the COTM sector and the relevant technical and administrative hurdles have been overcome, extending the performance envelope of COTM to include LEO satellites might be considered a next step. In addition, the significant regulatory activity aimed at allowing terrestrial 5G technologies to move into the spectrum used by FSS at C-band may present an opportunity to shift the focus of the FSS C-band market to supporting LEO satellite customers instead of Earth station customers.

Even if the rules are changed, there will still be some regulatory requirements for a LEO satellite to relay its communications through a GEO satellite. The recent FCC licensing of the commercial relay service started by Audacy includes a requirement that foreign registered customer satellites must apply for access to U.S. markets if the relay satellite downlinks the customer's traffic in the United States. This is not a difficult regulatory step but in a LEO to GEO satellite relay, a customer LEO satellite would likely have to register for access to all of the markets in which the GEO satellites that are used for the relay are based.

\section{CONCLUSIONS}

The regulatory hurdles are the biggest challenge to the successful implementation of this satellite communications option for LEO satellites. If experimental licenses can be secured that can help demonstrate the use of the FSSs for LEO satellite relay, then the satellite service providers and possibly a few governmental agencies may be willing to sponsor an effort to change the ITU rules regarding ISL in FSS spectrum. One scenario for such a test would be to place a modified FSS very small aperture terminal on the international space station and establish an FSS connection with several FSS GEO satellites and their Earth stations. Such a test would validate the concept of LEO satellites relaying through GEO satellites and provide a test bed to 
investigate the technical and administrative challenges associated with the solution.

To date, among the FSS vendors, only Viasat has published research on using existing FSS spectrum to support Ka band LEO to GEO satellite relays. Chandler, ${ }^{24}$ a Viasat antennas and communications systems architect, proposed a new hybrid network of high-rate LEO to Earth and LEO to GEO relays at Ka band, using the existing FSS Ka band spectrum for the LEO to GEO relays. However, the article does not address adapting the solution to the existing GEO FSS satellites. Currently, Viasat operates 3 high-capacity Ka-band-only GEO satellites.

There is an intriguing FSS LEO to GEO relay research project that has been started by the European Space Agency (ESA) Advanced Research in Telecommunications System program. ${ }^{25}$ In late 2018, the ESA solicited research proposals to investigate using C-band FSS satellites for low data rate ISL. This project has been funded and may yield some of the first real activity into developing a solution for utilizing the existing FSS satellite fleet to support LEO to GEO relay operations.

\section{ACKNOWLEDGMENTS}

The authors would like to thank the Federal Aviation Administration's Center of Excellence in Commercial Space Transportation and New Mexico State University who sponsored the study, as well as the University of North Florida who funded it.

\section{AUTHOR DISCLOSURE STATEMENT}

No competing financial interests exist.

\section{REFERENCES}

1. Space Works Enterprises. 2018 Nano/Microsatellite Market Forecast, 8th Edition. Atlanta, GA, 2018.

2. Frost \& Sullivan. Press release for market report entitled Small Satellite Services Market Quarterly Update 02 2018. August 7, 2018. www.prnewswire.com/newsreleases/disruptive-new-entrants-lead-small-satellite-launch-race-300692821 .html (Last accessed February 18, 2019).

3. Euroconsult. Press release for market report entitled Prospects for the Small Satellite Market. August 6, 2018. http://euroconsult-ec.com/6_August_2018 (Last accessed February 18, 2019).

4. Waltrop DW. Recovery of the Last GAMBIT and HEXAGON Film Buckets from Space, August-October 1984. Studies Intel. 2014; 58(2):19-34.

5. SpaceDaily.com. Laser Communications Demonstrated from CubeSats for the First Time. August 6, 2018. www.spacedaily.com/reports/Laser_Communicat ions_Demonstrated_From_Cubesats_For_The_First_Time_999.html (Last accessed February 18, 2019).

6. Gauthier S. Defense R\&D Canada, Operational Differences Between MEOSAR and GEO/LEOSAR Capabilities, Technical Memorandum DRDC CORA TM 2009011, 2009, Canada.

7. Israel DJ, Shaw H. Next-generation NASA Earth-orbiting relay satellites: Fusing optical and microwave communications. In: IEEE Aerospace Conference, Big Sky, Montana, March 2018.
8. Henry C. Commercial laser comm edges closer to reality, Spacenews.com. June 26, $2018 . \quad$ https://spacenews.com/commercial-laser-comm-edges-closer-toreality (Last accessed February 18, 2019).

9. Horan S. The Potential for using LEO telecommunications constellations to support nanosatellite formation flying. Int J Satellite Comms. 2002;20(5):347-61.

10. Zillig DJ, Perko KL, Nelson KG. New TDRSS communications options for small satellites. In: Proceedings of the 4th International Symposium on Space Mission Operations and Ground Data Systems, vol. 2, Munich, Germany, 1996:690-7.

11. NASA Space Communications and Navigation Deputy Program Manager for Operations, memo to: Goddard Space Flight Center Associate Director of Flight Projects, subject: Space Network Reimbursable for Fiscal Year 2017, October 1, 2016.

12. FCC International Bureau satellite license callsign S2982, file number SAT-LOA20161115-00117, issued June 4, 2018.

13. Reid D. Graduates of SpaceX, NASA, and Stamford are building an internet in space. August 10, 2018. www.cnbc.com/2018/08/10/using-spacex-and-nasagraduates-audacy-is-building-an-internet-in-spac.html (Last accessed February 18, 2019).

14. Audacy. Press released entitled Audacy Achieves $\$ 100$ Million in Commercial Agreements. October 1, 2018. https://audacy.space/press-releases-2/2018/9/27/ audacy-achieves-100-million-in-commercial-agreements (Last accessed February 18,2019$)$.

15. Werner D. Inmarsat and AVI's satellite data-relay service exits stealth mode after months of secret, in-space tests, Spacenews.com. February 22, 2017. https://spacenews.com/after-months-of-secret-in-space-testing-inmarsat-avissatellite-data-relay-service-exits-stealth-mode/ (Last accessed February 18, 2019).

16. Wengryniuk J. Comments of Inmarsat Inc., Filed in FCC International Bureau Docket 18-86. July 9, 2018. https://ecfsapi.fcc.gov/file/1070952424025/Inmar sat\%20Small\%20Sat\%20Comments \%20(final).pdf (Last accessed February 18, 2019).

17. Knogl JS. Geostationary Data Relays for Low Earth Orbit Satellites, Technical University of Munich, June 18, 2014, Published Dissertation for Ph.D.

18. Rao VS. Extend LEO Downlinks with GEO Satelllites, Microwaves \& RF, November 30, 2016. www.mwrf.com/print/9497

19. Satellite Industry Association. 2017 State of the Satellite Industry Report, June 2017.

20. IEEE Standard Letter Designations for Radar-Frequency Bands. In: IEEE Std 5212002 (Revision of IEEE Std 521-1984), 2003, DOI: 10.1109/IEEESTD.2003.94224

21. Baumgartner J. Viasat Downplays Antenna Issue Impacting ViaSat-2's Data Performance. February 15, 2018. www.multichannel.com/blog/viasat-downplaysantenna-issue-impacting-viasat-2-s-data-performance-418181 (Last accessed February 18, 2019).

22. Clark S. Ariane 5 rocket deploys satellites for Intelsat and B-SAT. September 29 , 2017. https://spaceflightnow.com/2017/09/29/ariane-5-rocket-deploys-satellitesfor-intelsat-and-b-sat/ (Last accessed February 18, 2019).

23. Gilat. Marketing brochure entitled Full In-Flight Satellite Connectivity Portfolio. December 18, 2016. www.gilat.com/news-resources/solution-resources/?resid= 1824 (Last accessed February 18, 2019).

24. Chandler B. A Ka-band Hybrid Ground/Space Communications Network for SmallSats, Space0p Conference, Marsellie, France, May 28-June 1, 2018.

25. European Space Agency Project: ARTES AT 5A.054, C-Band Intersatellite Link, Proposal tender number A09193.
Address correspondence to:

Brian Kopp

Electrical Engineering Department

University of North Florida

1 UNF Drive

Jacksonville, FL 32224

E-mail: brian.kopp@unf.edu 